\title{
On Projections in the Noncommutative 2-Torus Algebra ${ }^{\star}$
}

Michat ECKSTEIN

Faculty of Mathematics and Computer Science, Jagellonian University, ul. Eojasiewicza 6, 30-348 Kraków, Poland

E-mail: michal.eckstein@uj.edu.pl

Received December 09, 2013, in final form March 16, 2014; Published online March 23, 2014

http://dx.doi.org/10.3842/SIGMA.2014.029

\begin{abstract}
We investigate a set of functional equations defining a projection in the noncommutative 2-torus algebra $A_{\theta}$. The exact solutions of these provide various generalisations of the Powers-Rieffel projection. By identifying the corresponding $K_{0}\left(A_{\theta}\right)$ classes we get an insight into the structure of projections in $A_{\theta}$.
\end{abstract}

Key words: noncommutative torus; projections; noncommutative solitons

2010 Mathematics Subject Classification: 46L80; 19A13; 19K14; 46L87

\section{Introduction}

Projections (i.e. selfadjoint, idempotent elements) in associative $*$-algebras are the main building blocks of the algebraic $K$-theory. Commutative $C^{*}$-algebras, which by Gelfand-Naimark theorem are equivalent to locally compact Hausdorff spaces, do not contain non-trivial projections, when the corresponding space is connected. To determine the $K_{0}$ group of a unital $C^{*}$-algebra $\mathcal{A}$ one thus has to study the equivalence classes of projections in the matrix algebra $M_{\infty}(\mathcal{A})$. However, when one abandons the assumption of commutativity of the algebra one may encounter various non-trivial projections in the algebra itself which, in some cases, are sufficient to fully determine the group $K_{0}(\mathcal{A})$.

The $K$-theory of the noncommutative 2 -torus algebra $A_{\theta}$, known also as the irrational rotation algebra, has been thoroughly investigated in the 1980's. From the works of Pimsner, Voiculescu and Rieffel (see $[16,18]$ and references therein) we know that $K_{0}\left(A_{\theta}\right) \cong \mathbb{Z} \oplus \theta \mathbb{Z} \cong \mathbb{Z} \oplus \mathbb{Z}$. In the case of noncommutative tori it turns out that projections in the algebra $A_{\theta}$ itself generate the whole group $K_{0}\left(A_{\theta}\right)$ (see [19, Corollary 7.10]). The $K_{0}$ class of a projection is uniquely determined by its algebraic trace, so any two projections with the same trace must be unitarily equivalent in $M_{\infty}\left(A_{\theta}\right)$ (see [18, Corollary 2.5]). On the other hand, it has been already pointed out by Rieffel in [19] that the structure of projections in $A_{\theta}$ is more robust than it would appear from the $K$-theory level.

The purpose of this paper is to look closer into the structure of projections in $A_{\theta}$ itself. Our main results are summarised in Theorems 1 and 2 in Section 4. Proposition 3 addresses the problem of the existence of projections invariant under the flip automorphism (23). The statements are proven by an explicit construction of the relevant projections. The latter may be useful in the applications where explicit formulae for projections are needed. For an example see [8], where projections in $M_{2}\left(C\left(\mathbb{T}^{2}\right)\right)$ were constructed using Rieffel's method, which we generalise here ${ }^{1}$.

${ }^{\star}$ This paper is a contribution to the Special Issue on Noncommutative Geometry and Quantum Groups in honor of Marc A. Rieffel. The full collection is available at http://www.emis.de/journals/SIGMA/Rieffel.html

${ }^{1}$ We thank the anonymous referee for pointing out this possible application to us. 
The uses of noncommutative tori in physics are multifarious. A most natural one concerns gauge theories developed in terms of finitely generated projective modules, which are noncommutative counterparts of vector bundles $[4,21]$. Recently, the projections in the noncommutative torus algebra $A_{\theta}$ gained more interest in the context of string theory [5, 20]. They turned out to be extrema of the tachyonic potential providing solitonic field solutions interpreted in terms of D-branes $[1,12,13]$. Moreover, the projections in $A_{\theta}$ are extensively used in the context of quantum anomalies [6, 15], knot theory [9] or theoretical engineering [14].

The paper is organised as follows: Below we recall some basic definitions to fix notation and make the paper self-contained. In the next section we present a set of functional equations defining a projection in $A_{\theta}$ and comment on the adopted method of solving these. We also compute the Chern class of a projection satisfying these equations. In Section 3 we investigate some special solutions - the Powers-Rieffel type projections. These will serve us firstly to provide an alternative proof of the important Corollary 7.10 from [19]. Secondly, we will use them as a starting point for generalisations to come in Section 4. Section 5 contains the discussion of the flip-invariance (23). Finally, in Section 6, we make conclusions and discuss some open questions that arose during the presented analysis.

The algebra of noncommutative 2-torus $A_{\theta}$ is the universal $C^{*}$-algebra generated by two unitaries $U, V$ satisfying the following commutation relation

$$
V U=e^{2 \pi i \theta} U V
$$

for some real parameter $\theta \in[0,1[$, which we assume to be irrational.

We shall work with $\mathcal{A}_{\theta}$, a dense *-subalgebra [11,21] of $A_{\theta}$ which is made up of "smooth" elements of the form

$$
A_{\theta} \supset \mathcal{A}_{\theta} \ni a=\sum_{(m, n) \in \mathbb{Z}^{2}} a_{m, n} U^{m} V^{n}, \quad \text { with } \quad\left\{a_{m, n}\right\} \in \mathcal{S}\left(\mathbb{Z}^{2}\right),
$$

where $\mathcal{S}\left(\mathbb{Z}^{2}\right)$ denotes the space of Schwartz sequences on $\mathbb{Z}^{2}$, i.e.

$$
\left\{a_{m, n}\right\} \in \mathcal{S}\left(\mathbb{Z}^{2}\right) \Longleftrightarrow \sup _{m, n \in \mathbb{Z}}\left(1+m^{2}+n^{2}\right)^{k}\left|a_{m, n}\right|<\infty, \quad \text { for all } k \in \mathbb{N} .
$$

Let us note that $\mathcal{A}_{\theta}$ is a Fréchet pre- $C^{*}$-algebra [21], hence $K_{0}\left(\mathcal{A}_{\theta}\right) \cong K_{0}\left(A_{\theta}\right)$ as Abelian groups [10, Theorem 3.44].

For the purposes of this paper we will only consider elements of $\mathcal{A}_{\theta}$ with $a_{m, n} \neq 0$ for a finite range of the index $m$. These can be written as

$$
b=\sum_{m \text {-finite }} \sum_{n \in \mathbb{Z}} b_{m, n} U^{m} V^{n}, \quad \text { with } \quad\left\{b_{m, n}\right\}_{n \in \mathbb{Z}} \in \mathcal{S}(\mathbb{Z}) \quad \text { for any } m .
$$

Recall that any function $f \in C^{\infty}\left(S^{1}\right)$, regarded as a periodic function on $\mathbb{R}$ with period 1 , has a Fourier series presentation

$$
f(x)=\sum_{n \in \mathbb{Z}} f_{n} e^{2 \pi i x n}, \quad \text { with } \quad\left\{f_{n}\right\} \in \mathcal{S}(\mathbb{Z}) .
$$

Thus, via the funcional calculus, we may uniquely define an element from $\mathcal{A}_{\theta}$ by

$$
f(V)=\sum_{n \in \mathbb{Z}} f_{n} V^{n}, \quad \text { for } \quad f \in C^{\infty}\left(S^{1}\right)
$$

and the expression (3) is conveniently rewritten as

$$
b=\sum_{m \text {-finite }} U^{m} b_{m}(V), \quad \text { with } \quad b_{m} \in C^{\infty}\left(S^{1}\right) \quad \text { for any } m .
$$


The noncommutative 2-torus algebra is equipped with a canonical trace [11, 21], which on the elements of the form (4) can be expressed as (compare with [17, p. 415])

$$
\tau(b)=b_{0,0}=\int_{0}^{1} b_{0}(x) \mathrm{d} x .
$$

We shall use it to determine the $K_{0}$ class of a projection on the strength of Corollary 2.5 in [18].

To make a connection with the original framework of Rieffel's construction [17] we shall describe $A_{\theta}$ as the crossed product algebra $C\left(S^{1}\right) \rtimes \mathbb{Z}$. Here $\mathbb{Z}$ acts on $S^{1}$ by rotations by $2 \pi \theta$ and thus induces an action of $\mathbb{Z}$ as automorphisms of $C\left(S^{1}\right)$. A convenient concrete realisation of $A_{\theta}$ as bounded operators on $L^{2}\left(S^{1}\right)$ is obtained with

$$
(U f)(x)=f(x+\theta), \quad(V f)(x)=e^{2 \pi i x} f(x) .
$$

Note that the elements of the form (4) fall in the dense $*$-subalgebra of $A_{\theta}$ considered by Rieffel in [17]. The only difference is that we have chosen to work with the smooth functions on $S^{1}$ rather than continuous ones. This is necessary as we want to compute the Chern number [3] of the relevant projections, which requires $b_{n}$ functions to be differentiable.

Let $\delta_{1}, \delta_{2}$ be the basic unbounded derivations of $A_{\theta}$, which act on the generators as

$$
\delta_{1} U=2 \pi i U, \quad \delta_{1} V=0, \quad \delta_{2} U=0, \quad \delta_{2} V=2 \pi i V .
$$

Then the Chern number of a projection reads

$$
c_{1}(p)=\frac{1}{2 \pi i} \tau\left(p\left(\delta_{1} p \delta_{2} p-\delta_{2} p \delta_{1} p\right)\right) .
$$

The Chern number is related to the index of a Fredholm operator and thus it is always an integer (see [3, Theorem 11]).

\section{Equations for a projection in $\mathcal{A}_{\theta}$}

Having recalled the basic features of the noncommutative 2-torus algebra we are ready to investigate the structure of projections in it.

Let us consider the following element of $\mathcal{A}_{\theta}$ :

$$
p=\sum_{n=-M}^{M} U^{n} p_{n}(V), \quad \text { for some } \quad M \in \mathbb{N} .
$$

The conditions for $p$ to be a projection yield a set of functional equations for the functions $p_{i} \in C^{\infty}(\mathbb{R} / \mathbb{Z})$

$$
\begin{aligned}
& p_{k}(x)=\overline{p_{-k}(x+k \theta)}, \quad \text { for } \quad k=-M, \ldots, M, \\
& p_{k}(x)=\sum_{m, a=-M}^{M} p_{m}(x+a \theta) p_{a}(x) \delta_{m+a, k}, \quad \text { for } \quad k=-M, \ldots, M, \\
& 0=\sum_{m, a=-M}^{M} p_{m}(x+a \theta) p_{a}(x) \delta_{m+a, k}, \quad \text { for } \quad k<-M \quad \text { and } \quad k>M .
\end{aligned}
$$

Some of the above equations are redundant and the number of independent ones is $3 M+2$. It can be easily seen by noticing that equations (7)-(9) with $k<0$ are equivalent to those with $k>0$, because the functions $p_{k}$ with negative indices are actually defined by (7) with $k>0$. 
For $M=0$ formulae (7)-(9) imply $p_{0}(x) \equiv 1$ as one may expect. When $M=1$ one obtains the familiar Powers-Rieffel equations [17]. However, for $M \geq 2$ the equations become more and more involved and even the existence of a solution is not obvious. In [6] we found four particular solutions to (7)-(9) with $M=2$, which represent different classes of $K_{0}\left(\mathcal{A}_{\theta}\right)$. In the next sections we present a generalisation of the construction given in [6]. Before we start solving the equations (7)-(9) let us adopt the following definition.

Definition 1. We say a projection in $\mathcal{A}_{\theta}$ is of $\operatorname{order} M$ if it is of the form (6) and $p_{M} \neq 0$.

We shall not attempt to provide a general solution to (7)-(9), but rather present a class of special solutions. Nevertheless, this class turns out to be large enough to accommodate the known projections as well as a number of new ones.

We will consider only real-valued functions although (7) requires only $p_{0}$ to be real. Moreover, we have already noted that (7) defines the functions $p_{k}$ for $k<0$ and it is convenient to get rid of the functions $p_{k}$ with negative index $k$ in the equations (8) and (9) before solving them. Our special solutions will be such that each summand on the r.h.s. of (9) is equal to zero independently. The same should hold for summands of (8) with $k>0$ excluding those with $m=0$ or $a=0$, these are combined to form equations

$$
p_{k}(x)\left(p_{0}(x)+p_{0}(x+k \theta)-1\right)=0, \quad \text { for } \quad k=1, \ldots, M,
$$

which we also require to be satisfied independently. The equations (8) with $k<0$ are redundant and the case $k=0$ cannot be split into independent equations. After (7) is substituted into (8) for $k=0$ we obtain

$$
\begin{gathered}
p_{M}^{2}(x-M \theta)+p_{M}^{2}(x)+p_{M-1}^{2}(x-(M-1) \theta)+p_{M-1}^{2}(x) \\
+\cdots+p_{1}^{2}(x-\theta)+p_{1}^{2}(x)+p_{0}(x)\left(p_{0}(x)-1\right)=0 .
\end{gathered}
$$

In the forthcoming sections we provide a systematic method of constructing projections of a given trace (5) and order, that will satisfy the equations (7)-(9) refined according to the above-listed conditions.

Before we start, let us compute the Chern number of a projection of order $M$, as this quantity might prove useful in the task of classification.

Proposition 1. The Chern number of a projection $p$ of order $M$ reads

$$
\begin{aligned}
c_{1}(p)= & \sum_{n=1}^{M} \sum_{k=-M}^{M-n} \int_{0}^{1} \mathrm{~d} x\left(n \overline{p_{k+n}(x)}\left[p_{n}(x+k \theta) p_{k}^{\prime}(x)-p_{n}(x) p_{k}^{\prime}(x+n \theta)\right]\right. \\
& +(\{k, n\} \longleftrightarrow\{-k,-n\})) .
\end{aligned}
$$

Moreover, for any projection constructed with the method adopted in this paper, the formula (12) simplifies to

$$
c_{1}(p)=6 \int_{0}^{1} \mathrm{~d} x \sum_{n=1}^{M} n p_{n}(x)^{2} p_{0}^{\prime}(x) .
$$

Proof. The application of the derivations $\delta_{1}, \delta_{2}$ to a projection $p$ of the form (6) yields

$$
\delta_{1} p=2 \pi i \sum_{n=-M}^{M} n U^{n} p_{n}(V), \quad \delta_{2} p=\sum_{n=-M}^{M} U^{n} p_{n}^{\prime}(V) .
$$


Let us denote $p_{k}:=0$ for $|k|>M$. Then using (1), (5) and (7) we obtain

$$
\begin{aligned}
c_{1}(p) & =\sum_{j, k, n=-M}^{M} \tau\left(U^{j} p_{j}(V)\left[k U^{k} p_{k}(V) U^{n} p_{n}^{\prime}(V)-n U^{k} p_{k}^{\prime}(V) U^{n} p_{n}(V)\right]\right) \\
& =\sum_{j, k, n=-M}^{M} \tau\left(U^{j+k+n} p_{j}\left(e^{2 \pi i \theta(k+n)} V\right)\left[k p_{k}\left(e^{2 \pi i \theta n} V\right) p_{n}^{\prime}(V)-n p_{k}^{\prime}\left(e^{2 \pi i \theta n} V\right) p_{n}(V)\right]\right) \\
& =\sum_{k, n=-M}^{M} \int_{0}^{1} \mathrm{~d} x p_{-k-n}(x+(k+n) \theta)\left[k p_{k}(x+\theta n) p_{n}^{\prime}(x)-n p_{k}^{\prime}(x+n \theta) p_{n}(x)\right] \\
& =\sum_{k, n=-M}^{M} n \int_{0}^{1} \mathrm{~d} x \overline{p_{k+n}(x)}\left[p_{n}(x+\theta k) p_{k}^{\prime}(x)-p_{k}^{\prime}(x+n \theta) p_{n}(x)\right] .
\end{aligned}
$$

In the last equality we have relabelled the indices $k \leftrightarrow n$ in the first term in the square bracket. The formula (12) is just the above expression, with the fact $p_{k}=0$ for $|k|>M$ taken into account.

Now, in the method adopted in this paper we have assumed that the summands of the r.h.s. of (8) and (9) are equal to zero independently, excluding those with $m=0$ or $a=0$. This means that for the considered projections only the terms $k=0$ and $k=-n$ will contribute to the sum in (12) and we have

$$
\begin{aligned}
c_{1}(p)= & \sum_{n=1}^{M} \int_{0}^{1} \mathrm{~d} x n\left(p_{n}(x)^{2} p_{0}^{\prime}(x)-p_{n}^{2}(x) p_{0}^{\prime}(x+n \theta)+2 p_{0}(x) p_{n}(x-n \theta) p_{-n}^{\prime}(x)\right. \\
& \left.-2 p_{0}(x) p_{n}(x) p_{-n}^{\prime}(x+n \theta)+p_{n}(x-n \theta)^{2} p_{0}^{\prime}(x-n \theta)-p_{n}^{2}(x-n \theta) p_{0}^{\prime}(x)\right) \\
= & 2 \sum_{n=1}^{M} \int_{0}^{1} \mathrm{~d} x n\left(p_{n}(x)^{2} p_{0}^{\prime}(x)-p_{n}^{2}(x) p_{0}^{\prime}(x+n \theta)\right. \\
& \left.+p_{0}(x) p_{n}(x-n \theta) p_{n}^{\prime}(x-n \theta)-p_{0}(x) p_{n}(x) p_{n}^{\prime}(x)\right),
\end{aligned}
$$

We have used the formula (7) together with the assumption of all $p_{n}$ being real and the fact that shifting the integration variable does not change the value of the integral. Now, let us make use of the periodicity of the integrant to integrate by parts one of the terms and shift the variable in another:

$$
\begin{aligned}
c_{1}(p)= & 2 \sum_{n=1}^{M} \int_{0}^{1} \mathrm{~d} x n\left(p_{n}(x)^{2} p_{0}^{\prime}(x)+2 p_{n}(x) p_{n}^{\prime}(x) p_{0}(x+n \theta)\right. \\
& \left.+p_{0}(x+n \theta) p_{n}(x) p_{n}^{\prime}(x)-p_{0}(x) p_{n}(x) p_{n}^{\prime}(x)\right) .
\end{aligned}
$$

Let us note that equations (10) imply

$$
\begin{aligned}
\int_{0}^{1} \mathrm{~d} x p_{n}^{\prime}(x) p_{n}(x) p_{0}(x+n \theta) & =\int_{0}^{1} \mathrm{~d} x p_{n}^{\prime}(x) p_{n}(x)-\int_{0}^{1} \mathrm{~d} x p_{n}^{\prime}(x) p_{n}(x) p_{0}(x) \\
& =-\int_{0}^{1} \mathrm{~d} x p_{n}^{\prime}(x) p_{n}(x) p_{0}(x)
\end{aligned}
$$

since $p_{n}^{\prime}(x) p_{n}(x)$ is a total derivative. Finally, we obtain

$$
c_{1}(p)=2 \sum_{n=1}^{M} \int_{0}^{1} \mathrm{~d} x n\left(p_{n}(x)^{2} p_{0}^{\prime}(x)-4 p_{0}(x) p_{n}(x) p_{n}^{\prime}(x)\right)=6 \sum_{n=1}^{M} \int_{0}^{1} \mathrm{~d} x n p_{n}(x)^{2} p_{0}^{\prime}(x) .
$$




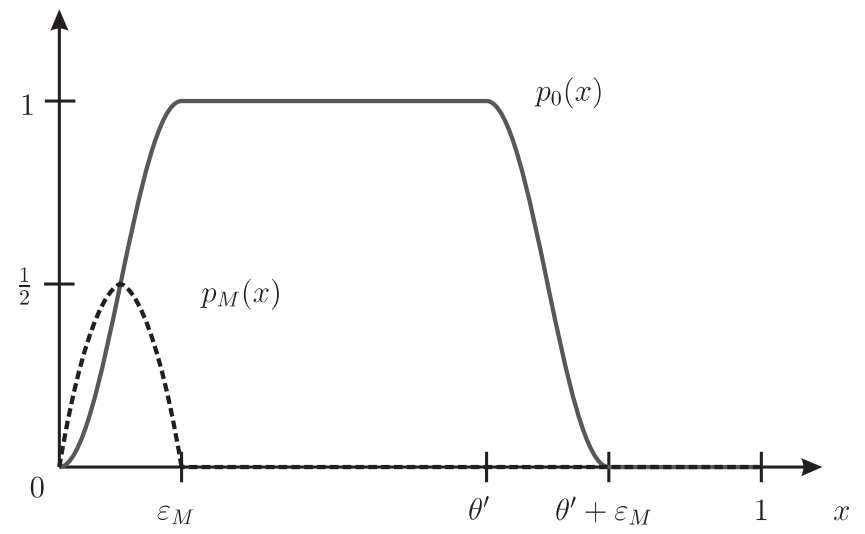

Figure 1. Depiction of functions constituing a Powers-Rieffel type projection. We have $0<\varepsilon_{M} \leq \theta^{\prime}$, $\varepsilon_{M}+\theta^{\prime} \leq 1$.

\section{Powers-Rieffel type projections}

We start with recalling the construction of the Powers-Rieffel projection in a slightly more general framework. It will serve us as a starting point for generalisations to come in the next section.

If one sets $p_{k}=0$ for all $1 \leq k \leq M-1$ then (7)-(9) reduce to the Powers-Rieffel equations with parameter $M \theta$ [17, Theorem 1.1]

$$
\begin{aligned}
& p_{M}(x+M \theta) p_{M}(x)=0, \\
& p_{M}^{2}(x)+p_{M}^{2}(x-M \theta)+p_{0}(x)\left(p_{0}(x)-1\right)=0, \\
& p_{M}(x)\left(1-p_{0}(x)-p_{0}(x+M \theta)\right)=0 .
\end{aligned}
$$

A standard solution to (14)-(16) is known as a Powers-Rieffel type projection [7, 13]

$$
\begin{gathered}
p_{0}(x)= \begin{cases}d_{M}(x), & 0 \leq x<\varepsilon_{M}, \\
1, & \varepsilon_{M} \leq x \leq M \theta, \\
1-d_{M}(x-M \theta), & M \theta \leq x<M \theta+\varepsilon_{M}, \\
0, & M \theta+\varepsilon_{M} \leq x \leq 1,\end{cases} \\
p_{M}(x)= \begin{cases}\sqrt{d_{M}(x)\left(1-d_{M}(x)\right)}, & 0 \leq x<\varepsilon_{M}, \\
0, & \varepsilon_{M}<x \leq 1,\end{cases}
\end{gathered}
$$

where $\theta^{\prime}=M \theta-\lfloor M \theta\rfloor$ and $d_{M}$ is a smooth function with $d_{M}(0)=0, d_{M}\left(\varepsilon_{M}\right)=1$. The functions $p_{0}$ and $p_{1}$ are depicted in Fig. 1.

Let us stress that we do not assume that the $d_{M}$ function starts growing directly at $x=0$ as shown on Fig. 1. We may take $d_{M}$ such that $d_{M}=0$ for $x \in\left[0, \delta_{M}\right]$ with some $\delta_{M}<\varepsilon_{M}$ and then smoothly growing to reach 1 at $x=\varepsilon_{M}$. This ensures that what we call here a PowersRieffel type projection is sufficiently general to incorporate the existing definitions (see [13] for instance).

Let us now discuss the properties of these projections. First of all, note that due to the periodicity of $p_{i}$ functions, equations (14)-(16) are invariant with respect to the transformation $M \theta \rightarrow M \theta+z$ for any $z \in \mathbb{Z}$. This means that a Powers-Rieffel type projection of order $M$ has the algebraic trace (5) equal to $\theta^{\prime}=M \theta-\lfloor M \theta\rfloor$. Since $\theta$ is irrational we have infinitely many $M$ such that

$$
0<M \theta-n<1 \Longleftrightarrow \frac{n}{M}<\theta<\frac{n+1}{M} .
$$


Hence, the following proposition (which is also a consequence of the Corollary 7.10 in [19]) holds.

Proposition 2. The algebra $\mathcal{A}_{\theta}$ contains projections representing infinitely many different classes of $K_{0}\left(\mathcal{A}_{\theta}\right)$.

Another point of view one may adopt for the projection (17)-(18) is that for any fixed $M$ it is the standard Powers-Rieffel projection [17] in the subalgebra of $\mathcal{A}_{\theta}$ generated by $U^{M}$ and $V$. This fact may be used to construct an approximation of $\mathcal{A}_{\theta}$ in terms of two algebras of matrix valued functions on $S^{1}[7,13]$.

For $p^{[M]}$ a Powers-Rieffel type projection of order $M$ the formula (13) gives

$$
c_{1}\left(p^{[M]}\right)=6 M \int_{0}^{\varepsilon_{M}} \mathrm{~d} x d_{M}(x)\left(1-d_{M}(x)\right) d_{M}^{\prime}(x)=\left.6 M\left(\frac{d_{M}(x)^{2}}{2}-\frac{d_{M}(x)^{3}}{3}\right)\right|_{0} ^{\varepsilon_{M}}=M .
$$

This is in accordance with the result of [3] stating that if $\tau(p)=|a-b \theta|$ then $c_{1}(p)= \pm b$.

From the $K$-theoretic point of view, these projections are sufficient to understand the structure of the equivalence classes of projective modules over $\mathcal{A}_{\theta}$. On the other hand, the algebra $\mathcal{A}_{\theta}$ contains other interesting projections, which we shall present in next section.

\section{More general projections in $\mathcal{A}_{\theta}$}

Let us now see what kind of projections one can get by letting functions $p_{k}$ in (6) to be nonzero for some of the indices $k \in\{1, \ldots, M-1\}$. The results are summarised in the following Theorems.

Theorem 1. A projection of order $M$ may represent the $K_{0}\left(\mathcal{A}_{\theta}\right)$ class $[n \theta]$, as well as the class $[1-n \theta]$, for all $n=1,2, \ldots, \frac{1}{2} M(M+1)$, provided that $0<\theta<1 / \max (n, M)$.

By $[n \theta] \in K_{0}\left(\mathcal{A}_{\theta}\right)$ we denote the $K_{0}$ class represented by a projection $p \in \mathcal{A}_{\theta}$ with $\tau(p)=n \theta$.

Theorem 2. The equations (7)-(9) for a projection of order $M$ admit solutions with $p_{k} \neq 0$ for every $k \in\{0, \ldots, M\}$ whenever $0<\theta<1 / M$.

We shall start with the proof of Theorem 1 by showing how to use the functions $p_{k}$ to increase or decrease the trace of a Powers-Rieffel type projection. Then we present a method of including the remaining $p_{k}$ functions into the projections constructed in the previous proof without changing its traces. In this way we will prove Theorem 2. Both proofs are constructive so we are able to plot some examples of the $p_{0}$ functions of the relevant projections which, as we shall see, determine all of the other functions $p_{k}$ for $k \neq 0$. A brief discussion of the assumptions limiting the $\theta$ parameter may be found in Section 6 .

Proof of Theorem 1. Let us start with the case of $\tau(p)=n \theta>M \theta$. We shall begin with a Powers-Rieffel type projection as defined in (17)-(18). First note that if $M \theta<1$ then the functions $p_{0}$ and $p_{M}$ of the Powers-Rieffel type projection of order $M$ vanish for $x \geq M \theta+\varepsilon_{M}$. If $\theta$ is small enough (i.e. $(M+k) \theta<1$ ) then we can "glue" a Powers-Rieffel type projection of trace $k \theta$ to the previous one. Namely, let us keep the definition of $p_{0}$ on $\left[0, M \theta+\varepsilon_{M}\right]$ (see (17)) and set

$$
p_{0}(x)= \begin{cases}d_{k}(x), & M \theta+\varepsilon_{M} \leq x<M \theta+\varepsilon_{M}+\varepsilon_{k}, \\ 1, & M \theta+\varepsilon_{M}+\varepsilon_{k} \leq x \leq(M+k) \theta+\varepsilon_{M}, \\ 1-d_{k}(x-k \theta), & (M+k) \theta+\varepsilon_{M} \leq x<(M+k) \theta+\varepsilon_{M}+\varepsilon_{k}, \\ 0, & (M+k) \theta+\varepsilon_{M}+\varepsilon_{k} \leq x \leq 1\end{cases}
$$



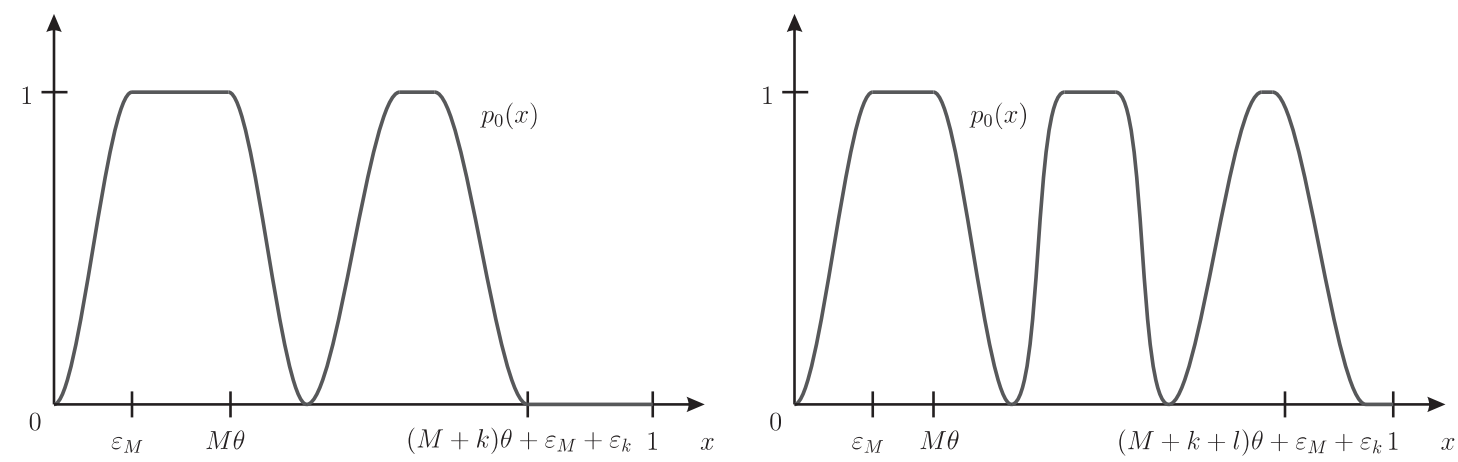

Figure 2. Examples of $p_{0}$ functions for projections with traces $(M+k) \theta$ and $(M+k+l) \theta$.

$$
p_{k}(x)= \begin{cases}\sqrt{d_{k}(x)\left(1-d_{k}(x)\right)}, & M \theta+\varepsilon_{M} \leq x<M \theta+\varepsilon_{M}+\varepsilon_{k}, \\ 0, & \text { elsewhere }\end{cases}
$$

for a smooth function $d_{k}$ with $d_{k}\left(M \theta+\varepsilon_{M}\right)=0, d_{k}\left(M \theta+\varepsilon_{M}+\varepsilon_{k}\right)=1$ and a small parameter $\varepsilon_{k}$. The summands of (8) and (9), which we have assumed to be equal to zero independently, have the form $p_{m}(x+a \theta) p_{a}(x)$. This means that all of the non-zero functions $p_{k}$ for $k \neq 0$ shifted to the interval $x \in[0, \theta]$ must not intersect. The latter can be fulfilled by restricting the parameters $\varepsilon$ such that

$$
0<\varepsilon_{M} \leq M \theta, \quad 0<\varepsilon_{k} \leq k \theta, \quad \varepsilon_{M}+\varepsilon_{k}+(M+k) \theta \leq 1
$$

implying that equation (11) reduces to two equations of the form (15). Namely for $x \in\left[0, \varepsilon_{M}\right] \cup$ $\left[M \theta, M \theta+\varepsilon_{M}\right]$ and for $x \in\left[M \theta+\varepsilon_{M}, M \theta+\varepsilon_{M}+\varepsilon_{k}\right] \cup\left[(M+k) \theta+\varepsilon_{M},(M+k) \theta+\varepsilon_{M}+\varepsilon_{k}\right]$ we have respectively

$$
\begin{aligned}
& p_{0}(x)\left(1-p_{0}(x)\right)=p_{M}^{2}(x)+p_{M}^{2}(x-M \theta), \\
& p_{0}(x)\left(1-p_{0}(x)\right)=p_{k}^{2}(x)+p_{k}^{2}(x-k \theta) .
\end{aligned}
$$

These equations are satisfied by the construction of $p_{k}$ and $p_{M}$. On the remaining part of the interval $[0,1]$ the equation (11) is trivially satisfied, since both l.h.s. and r.h.s. are equal to 0 . By the same argument, equation (10) remains satisfied, as it is satisfied for both Powers-Rieffel type projections independently. Thus, we have obtained a new projection with a trace $(M+k) \theta$. Examples of $p_{0}$ functions defining such projections are depicted in Fig. 2.

If the parameter $\theta$ is small enough (i.e. $n \theta<1$ ) we can continue the process of "glueing" Powers-Rieffel type projections to obtain a projection of trace $n \theta$, with $n \geq M$. If one makes use of all of the functions $p_{k}$ with $1 \leq k \leq M-1$ to increase the trace, one will end with a projection bearing the trace $(1+2+\cdots+M) \theta=\frac{1}{2} M(M+1) \theta$. The only thing one has to take care of are the conditions satisfied by the parameters $\varepsilon_{k}$. The restrictions (19) may be easily generalised to the case of non-vanishing $p_{k_{s}}$ functions with $s \in[1, M-1]$ :

$$
\begin{aligned}
& 0<\varepsilon_{k_{j}} \leq k_{j} \theta, \quad \text { for } \quad 1 \leq j \leq s, \\
& \varepsilon_{k_{1}}+\cdots+\varepsilon_{k_{s}}+\varepsilon_{M}+n \theta \leq 1, \quad \text { with } \quad n=k_{1}+\cdots+k_{s}+M .
\end{aligned}
$$

Let us note, that the above construction can be obtained (for $n \theta<1$ ) by taking a sum of $s$ mutually orthogonal Powers-Rieffel type projections $p^{\left[k_{j}\right]}$ of respective orders $k_{j}$. Indeed, one can easily check that the functional equations resulting form the projection and orthogonality conditions

$$
\left(p^{\left[k_{i}\right]}\right)^{2}=\left(p^{\left[k_{i}\right]}\right)^{*}=p^{\left[k_{i}\right]}, \quad p^{\left[k_{i}\right]} p^{\left[k_{j}\right]}=p^{\left[k_{j}\right]} p^{\left[k_{i}\right]}=0, \quad \text { for } \quad 1 \leq i \neq j \leq s,
$$



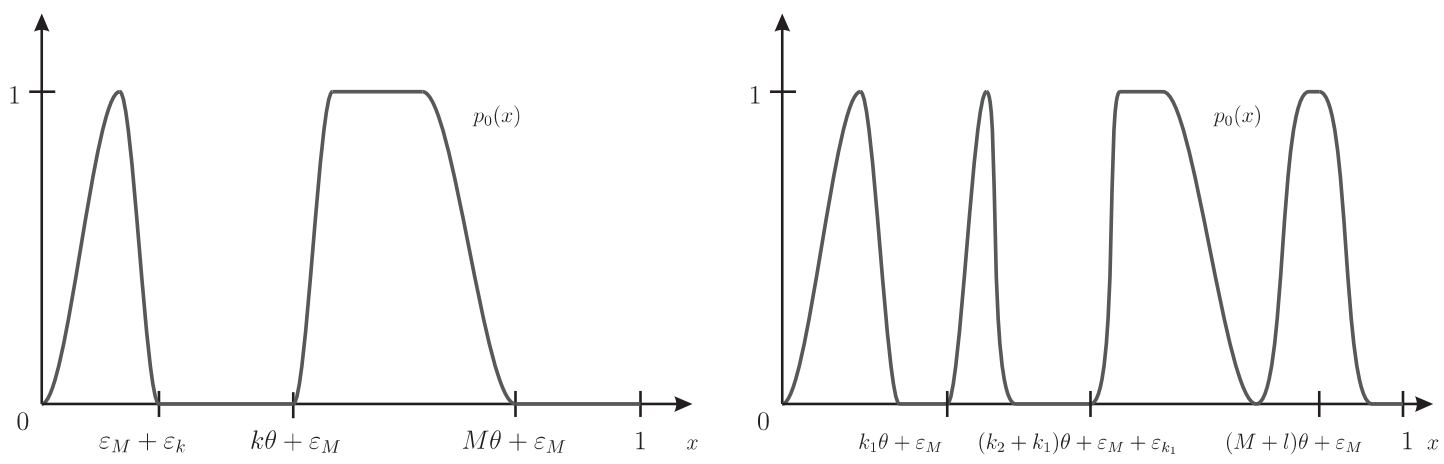

Figure 3. Examples of $p_{0}$ functions for projections of trace $(M-k) \theta$ and $\left(M-k_{1}-k_{2}+l\right) \theta$.

coincide with the ones derived in Section 2. This process of "glueing" mutually orthogonal Powers-Rieffel type projections appeared already in [7] and was extensively used therein. It has also been presented in [1] in a more similar form to the one shown above.

Let us now consider the case of projections of order $M$ and trace $n \theta$ with $1 \leq n<M$. Again, we shall use as a starting point a Powers-Rieffel type projection (17)-(18), but now we will "cut out" a part of it. Let us set

$$
\begin{aligned}
& p_{0}(x)= \begin{cases}d_{k}(x), & \varepsilon_{M} \leq x<\varepsilon_{M}+\varepsilon_{k}, \\
0, & \varepsilon_{M}+\varepsilon_{k} \leq x \leq k \theta+\varepsilon_{M}, \\
1-d_{k}(x-k \theta), & k \theta+\varepsilon_{M} \leq x<k \theta+\varepsilon_{M}+\varepsilon_{k}, \\
1, & k \theta+\varepsilon_{M}+\varepsilon_{k} \leq x \leq M \theta,\end{cases} \\
& p_{k}(x)= \begin{cases}\sqrt{d_{k}(x)\left(1-d_{k}(x)\right)}, & \varepsilon_{M} \leq x<\varepsilon_{M}+\varepsilon_{k}, \\
0, & \text { elsewhere }\end{cases}
\end{aligned}
$$

with a smooth function $d_{k}$ such that $d_{k}\left(\varepsilon_{M}\right)=1, d_{k}\left(\varepsilon_{M}+\varepsilon_{k}\right)=0$. The conditions $0<\varepsilon_{M} \leq M \theta$, $0<\varepsilon_{k} \leq k \theta$ and $\varepsilon_{M}+M \theta \leq 1$ should be satisfied. The situation is now completely analogous to the case of "glued" projections and the same arguments apply. A projection obtained in this way bears the trace $(M-k) \theta$ for $1 \leq k \leq M-1$ (see Fig. 3).

To end the proof of Theorem 1 it remains just to recall that if $p$ is a projection then obviously $1-p$ is so. This means that all of the considerations hold for projections of traces $(1-n \theta)$, one simply should take $1-p_{0}$ instead of $p_{0}$ and leave $p_{k}$ for $k \neq 0$ as they are.

The presented proof provides a great variety of possible projections with a given trace, which have, in general, different orders. Let us notice that the two procedures of increasing and decreasing the trace of a projection of a given order can be applied simultaneously and in arbitrary sequence (see Figs. 3 and 5). One only has to choose well the parameters $\varepsilon_{k}$ to have the equations (20) satisfied. These equations guarantee that the functions $d_{k}$ do not superpose and the equations (7)-(9) remain satisfied. This leads to an enormous number of projections if the order $M$ is big enough. Let us now pass on to the most general projections we were able to construct with the adopted method.

Proof of Theorem 2. In fact one can let all $p_{k}$ functions to be non-zero by incorporating to $p_{0}$ some "bump functions" $d_{k}$. As a starting point, one should take an arbitrary projection defined in Section 3 or 4 . For sake of simplicity let us now denote by $k$ a free index, i.e. we have $p_{k}=0$ in our starting point projection. Now, if one sets $p_{0}(x)=d_{k}(x)$ for $x \in\left[\delta_{k}, \delta_{k}+\varepsilon_{k}\right]$, with $d_{k}\left(\delta_{k}\right)=d_{k}\left(\delta_{k}+\varepsilon_{k}\right)=1$ or $d_{k}\left(\delta_{k}\right)=d_{k}\left(\delta_{k}+\varepsilon_{k}\right)=0$ then, to fulfil the equation (10), one has to set $p_{0}(x)=1-d_{k}(x-k \theta)$ for $x \in\left[k \theta+\delta_{k}, k \theta+\delta_{k}+\varepsilon_{k}\right]$. The function $p_{k}$ should then be defined 

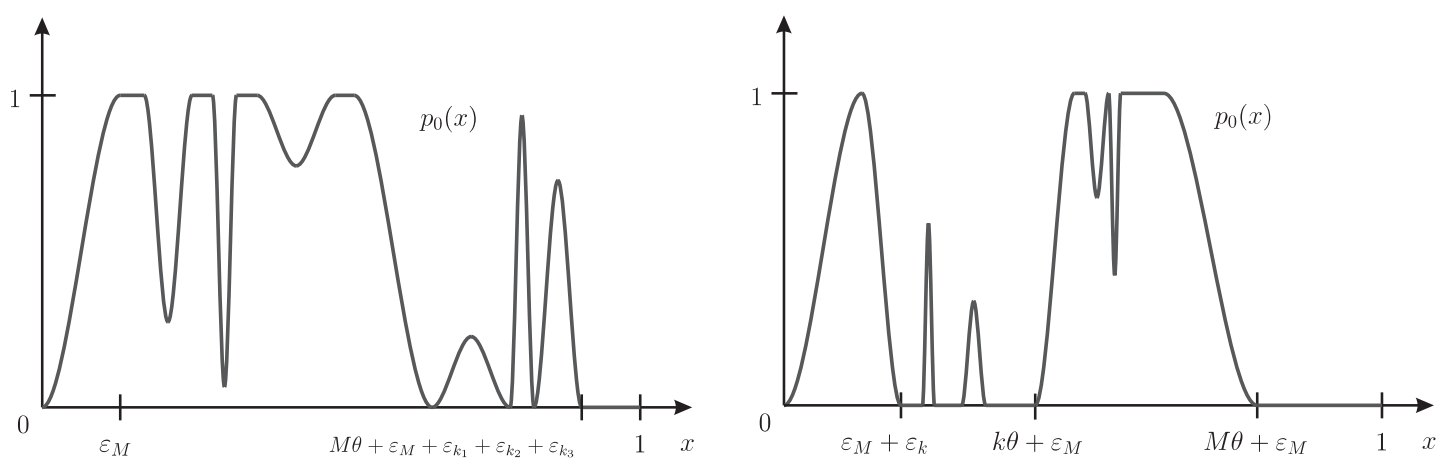

Figure 4. Examples of $p_{0}$ functions for projections of traces $M \theta$ and $(M-k) \theta$.

as previously by $\sqrt{d_{k}(x)\left(1-d_{k}(x)\right)}$ for $x \in\left[\delta_{k}, \delta_{k}+\varepsilon_{k}\right]$ and 0 elsewhere, so that (11) remains fulfilled. The only task to accomplish is to choose well the parameters $\varepsilon_{k}$ and $\delta_{k}$ to avoid the possible intersection of $d_{k}$ functions. The parameters $\varepsilon_{k}$ should be such that the equations (20) remain satisfied, and $\delta_{k}=n \theta+\varepsilon_{k_{1}}+\cdots+\varepsilon_{k_{s}}$ for $n, s \in \mathbb{Z}$ which depend on the concrete projection one has chosen as a starting point.

Examples of $p_{0}$ functions of the described above projections are shown in Fig. 4.

By giving constructive proofs of Theorems 1 and 2 we have exhausted all of the possibilities of constructing projections in $\mathcal{A}_{\theta}$ with the method described in Section 2. To end this section let us note that the computation of the Chern number of the newly constructed projections does not provide any new information. Indeed, it is straightforward either from direct computations of the formula (13), either from an application of the results of [3] that if we have a projection $p$ of trace $n \theta$, then $c_{1}(p)=n$. In particular, the process of adding "bump" functions described in the proof of Theorem 2 does not change the Chern class of a projection.

\section{$5 \quad$ Flip-symmetric projections}

The Powers-Rieffel type projection can be made invariant under the flip automorphism

$$
\sigma \in \operatorname{Aut} A_{\theta}, \quad \sigma(U)=U^{-1}, \quad \sigma(V)=V^{-1} .
$$

This is accomplished by setting $\varepsilon_{M}=1-M \theta$ and requiring that $d_{M}(x)+d_{M}\left(\varepsilon_{M}-x\right)=1$ for $x \in\left[0, \varepsilon_{M}\right]$ in formulae (17)-(18). It is interesting to check for which of the more general projections presented in this paper the flip symmetry can be imposed ${ }^{2}$.

Requiring $\sigma(p)=p$ for projections of order $M$ translates to the following constraints on functions $p_{k}$ :

$$
p_{0}(x)=p_{0}(1-x), \quad p_{k}(1-x)=\overline{p_{k}(x-k \theta)}, \quad \text { for } \quad k=1, \ldots, M .
$$

The fact that $p_{0}$ is periodic with period 1 implies that for a flip-symmetric projection, $p_{0}$ should be symmetric around $\frac{1}{2}$.

Let us first note that the flip symmetry cannot be imposed on a projection, which is "glued" from two or more segments (see Fig. 2). This is because the segments will necessarily have different lengths and thus $p_{0}$ cannot be symmetric around $\frac{1}{2}$. It is also clear (see Fig. 4) that the inclusion of a "bump function" breaks the flip invariance of a projection.

On the other hand, the "cutting out" procedure (see Fig. 3) can be performed in such a way that the invariance under the flip automorphism is preserved. Moreover, provided that the $\theta$

\footnotetext{
${ }^{2}$ We are grateful to the anonymous referee for suggesting this interesting problem to us.
} 

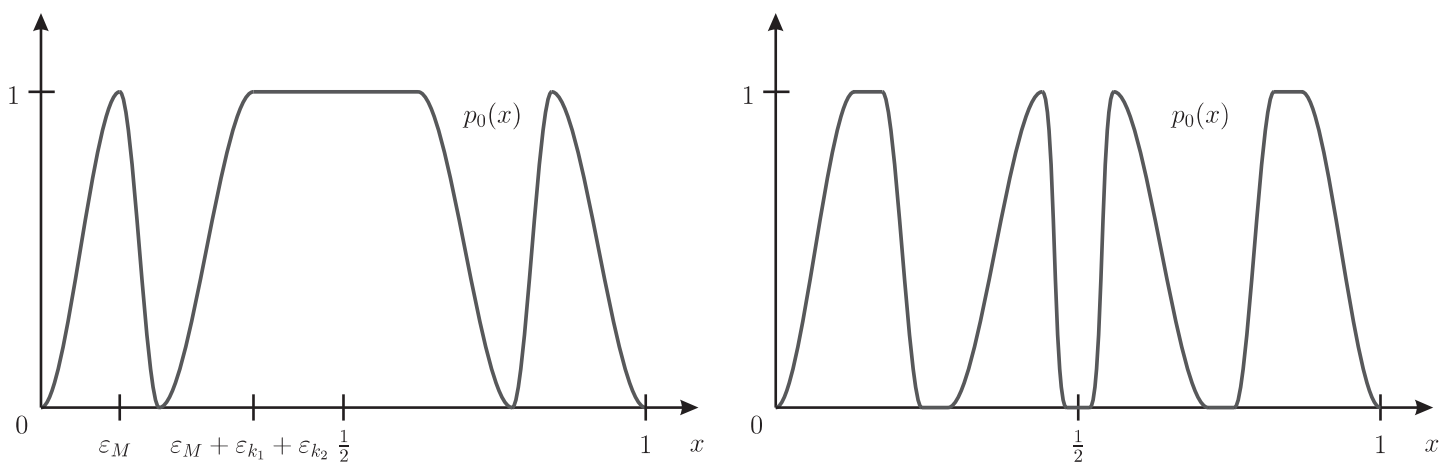

Figure 5. Examples of $p_{0}$ functions for flip-symmetric projections.

parameter is small enough, one can "glue" another Powers-Rieffel type projection inside the "cut-out" region in a symmetric way. This process may be continued as long as the parameter $\theta$ allows it. However, this requires a fine tuning of $\varepsilon_{k}$ parameters. Examples of $p_{0}$ functions of such flip-symmetric projection are depicted in Fig. 5.

Let us now formulate the above considerations in a precise way.

Proposition 3. Let $k_{j} \in\{1, \ldots, M-1\}$ for $j=1,2, \ldots, s, 1 \leq s \leq M-1$ be such that

$$
\left.\left.M>k_{1}>k_{2}>\cdots>k_{s}>0 \quad \text { and } \quad \theta \in\right] c_{1}^{-}, c_{1}^{+}\right] \cap\left[c_{2}^{-}, c_{2}^{+}\left[\cap \cdots \cap\left[c_{s}^{-}, c_{s}^{+}\right] \backslash\left\{c_{s}^{ \pm}\right\},\right.\right.
$$

where $\pm=(-1)^{s}$ and

$$
\begin{aligned}
& c_{j}^{+}= \begin{cases}\frac{1}{2\left(M-k_{1}+k_{2}-k_{3}+\cdots-k_{j}\right)}, & \text { for } j \text { odd }, \\
\frac{1}{2\left(M-k_{1}+k_{2}-k_{3}+\cdots-k_{j-1}\right)+k_{j}}, & \text { for } j \text { even, }\end{cases} \\
& c_{j}^{-}= \begin{cases}\frac{1}{2\left(M-k_{1}+k_{2}-k_{3}+\cdots+k_{j-1}\right)-k_{j}}, & \text { for } j \text { odd }, \\
\frac{1}{2\left(M-k_{1}+k_{2}-k_{3}+\cdots+k_{j}\right)}, & \text { for } j \text { even } .\end{cases}
\end{aligned}
$$

Then, there exists a projection $p \in \mathcal{A}_{\theta}$ of order $M$ invariant under the flip automorphism (23) $\sigma(p)=p$. Moreover, $p$ is a representant of the $K_{0}$ class $\left[\left(M-k_{1}+k_{2}-k_{3}+\cdots+(-1)^{s} k_{s}\right) \theta\right]$.

Proof. The construction of the projection $p$ goes as follows: We start with a Powers-Rieffel type projection of order $M(17)-(18)$. We make it flip symmetric, i.e. we set $\varepsilon_{M}=1-M \theta$ and require that $d_{M}$ be such that $d_{M}(x)+d_{M}\left(\varepsilon_{M}-x\right)=1$ for $x \in\left[0, \varepsilon_{M}\right]$. Then, we "cut out" a projection of trace $k_{1} \theta$ (see formulae (21)-(22)) also in symmetry-preserving way. This requires setting (compare the left plots in Figs. 3 and 5)

$$
\varepsilon_{k_{1}}=\left(2 M-k_{1}\right) \theta-1 \quad \text { and } \quad d_{k_{1}}(x)+d_{k_{1}}\left(\varepsilon_{k_{1}}-x\right)=1, \quad \text { for } \quad x \in\left[\varepsilon_{M}, \varepsilon_{M}+\varepsilon_{k_{1}}\right] .
$$

This choice guarantees that constraints (24) are fulfilled for both functions $p_{0}$ and $p_{k_{1}}$.

Note however, that we need to have $0<\varepsilon_{k_{1}} \leq k_{1} \theta$ for $p$ to be a projection, which is equivalent to

$$
\left.\left.\frac{1}{2 M-k_{1}}<\theta \leq \frac{1}{2\left(M-k_{1}\right)} \Longleftrightarrow \theta \in\right] c_{1}^{-}, c_{1}^{+}\right] .
$$

Now, we "glue" a Powers-Rieffel type projection of trace $k_{2} \theta$ in the middle of the "cut-out" region. To preserve the flip symmetry we have to set

$$
\varepsilon_{k_{2}}=1-2\left(M-k_{1}\right) \theta+k_{2} \theta
$$


and

$$
d_{k_{2}}(x)+d_{k_{2}}\left(\varepsilon_{k_{2}}-x\right)=1, \quad \text { for } \quad x \in\left[\varepsilon_{M}+\varepsilon_{k_{1}}, \varepsilon_{M}+\varepsilon_{k_{1}}+\varepsilon_{k_{2}}\right] .
$$

But since $0<\varepsilon_{k_{2}} \leq k_{2} \theta$, the equation on $\varepsilon_{k_{2}}$ can be fulfilled only if

$$
\frac{1}{2\left(M-k_{1}+k_{2}\right)} \leq \theta<\frac{1}{2\left(M-k_{1}\right)+k_{2}} \quad \Longleftrightarrow \quad \theta \in\left[c_{2}^{-}, c_{2}^{+}[.\right.
$$

By performing further consecutive "cut-outs" and "glueings" we obtain the following conditions for all $j \in\{1, \ldots, s\}$

$$
\begin{aligned}
& \varepsilon_{k_{j}}=\left\{\begin{array}{l}
2\left(M-k_{1}+k_{2}-k_{3}+\cdots-k_{j-1}\right) \theta-k_{j} \theta-1, \quad \text { for } j \text { odd, } \\
1-2\left(M-k_{1}+k_{2}-k_{3}+\cdots-k_{j-1}\right) \theta-k_{j}, \quad \text { for } j \text { even, }
\end{array}\right. \\
& d_{k_{j}}(x)+d_{k_{j}}\left(\varepsilon_{k_{j}}-x\right)=1, \quad \text { for } \quad x \in\left[\varepsilon_{M}+\varepsilon_{k_{1}}+\cdots+\varepsilon_{k_{j-1}}, \varepsilon_{M}+\varepsilon_{k_{1}}+\cdots+\varepsilon_{k_{j}}\right],
\end{aligned}
$$

which can be met only if $\theta \in\left[c_{j}^{-}, c_{j}^{+}\right] \backslash\left\{c_{j}^{ \pm}\right\}$, with $\pm=(-1)^{j}$.

To conclude the proof let us remind the reader that the procedure of "glueing" a projection of trace $k \theta$ increases the trace of the overall projection by $k \theta$ and "cutting-out" decreases it by $k \theta$.

We end this section with a remark, that some of the presented symmetric projections cannot be constructed in any $\mathcal{A}_{\theta}$ with $\theta \in\left[0,1\left[\right.\right.$. Let us take for instance $M=6, k_{1}=5, k_{2}=4, k_{3}=1$. Then $c_{1}^{-}=\frac{1}{7}, c_{1}^{+}=\frac{1}{2}, c_{3}^{-}=\frac{1}{9}, c_{3}^{+}=\frac{1}{8}$, so $\left.\left.\left.] c_{1}^{-}, c_{1}^{+}\right] \cap\right] c_{3}^{-}, c_{3}^{+}\right]=\varnothing$.

\section{Conclusion and open questions}

Let us now summarise the obtained results and outline the directions of possible further investigations.

We have presented many projections, which generalise the standard Powers-Rieffel projection. Some represented the same $K_{0}\left(\mathcal{A}_{\theta}\right)$ class, but had different orders. The others, conversely, had the same order, but different traces. A natural question one can ask is what are the relations between the presented projections? The answer is provided by Theorem 8.13 in [19]. It states that if two projections in $\mathcal{A}_{\theta}$ represent the same $K_{0}\left(\mathcal{A}_{\theta}\right)$ class (hence have the same trace), then, not only they are unitarily equivalent in $M_{\infty}\left(\mathcal{A}_{\theta}\right)$, but they are actually in the same path component of the set of projections in $\mathcal{A}_{\theta}$ itself. This means that there exists a homotopy of projections in $\mathcal{A}_{\theta}$ for any two projections which have the same trace. Indeed, if, for instance, one takes $d_{k}(t, x):=t d_{k}(x)+(1-t)$ instead of $d_{k}(x)$ with $d_{k}\left(\delta_{k}\right)=d_{k}\left(\delta_{k}+\varepsilon_{k}\right)=1$ for the "bump function" used in the proof of Theorem 2 , then one would obtain a projection for all $t \in[0,1]$.

In consequence, from the topological point of view it is sufficient to consider Powers-Rieffel type projections, since they are the generators of the $K_{0}\left(\mathcal{A}_{\theta}\right)$ group. On the other hand, the richness of the structure of projections may show up in applications. In the proof of the Theorem 1 it has already been mentioned that the procedure of "gluing" the Powers-Rieffel type projections is in fact equivalent to taking sums of mutually orthogonal projections. However, the "cutting out" described subsequently does not admit an interpretation in terms of subtracting the projections. Indeed, it is straightforward to see that if one expresses a projection $p$ of order $M$ and trace $(M-k) \theta$ as $p=q-r$, where $q$ is a Powers-Rieffel type projection of order $M$, then $r$ would not be a projection. This shows that the newly found projections are not just linear combinations of the $K_{0}$ generators.

The method adopted in this paper clearly does not pretend to cover every possible projection in $\mathcal{A}_{\theta}$. For a most general projection in $\mathcal{A}_{\theta}$ one would need to allow the order $M$ of a projection 
go to infinity. This would imply the need of working directly with the elements of the form (2) and would require completely different methods (see [2] for an example).

The puzzling thing about the newly found projections is that their existence in $\mathcal{A}_{\theta}$ seems to depend on the noncommutativity parameter $\theta$ as stated in the theorems in Section 4 . Unfortunately, the solutions presented there cannot be adapted to the case $n \theta>1$, as it was done for the Powers-Rieffel type projections in Section 3. It is so because the translational symmetry of (14)-(16), used in the proof of Proposition 2 is absent in general equations (7)-(9). Note that the discussed symmetry is also broken whenever we introduce the mentioned "bump functions". What is more, the existence of flip-symmetric projections in $\mathcal{A}_{\theta}$ seems to depend on $\theta$ in an even more peculiar way. Whether there is a true difference in the structure of projections in $\mathcal{A}_{\theta}$ depending on the noncommutativity parameter $\theta$ or is it just an artefact of our method of solving the equations (7)-(9) remains an open question.

To conclude the paper, let us comment on the possible applications of the obtained results to the $D$-brane scenario in Type II string theories. As mentioned in the Introduction, projections in $\mathcal{A}_{\theta}$ correspond to solitonic field configurations which are identified with $D$-branes $[1,12,13]$. On one hand, unitarly equivalent projections yield gauge equivalent field configurations [13, Section 3.1], hence the knowledge of $K_{0}\left(\mathcal{A}_{\theta}\right)$ alone seems to be sufficient. On the other hand, projections which cannot be written as linear combinations of $K_{0}$ generators provide non-perturbative field configurations. Moreover, the homotopy equivalence of projections may be exploited to study the soliton dynamics. An example is provided in [13, Section 6.2], where the Boca projection [2], which is homotopy equivalent to the standard Powers-Rieffel projection, is used. The possibility of adding "bump functions" to a projection as described at the end of Section 4 indicates the existence of an additional degree of freedom of the D-branes. It would also be interesting to investigate the consequences for $D$-branes of a projection being invariant under the flip symmetry. Finally, let us note that the $D$-brane point of view suggests that the number of projections in $\mathcal{A}_{\theta}$ indeed depends on the value of the deformation parameter $\theta$ (see [12, Section 4] or [1, Section V]).

\section{Acknowledgements}

We would like to thank Andrzej Sitarz for his illuminating remarks. Project operated within the Foundation for Polish Science IPP Programme "Geometry and Topology in Physical Models"

co-financed by the EU European Regional Development Fund, Operational Program Innovative Economy 2007-2013.

\section{References}

[1] Bars I., Kajiura H., Matsuo Y., Takayanagi T., Tachyon condensation on a noncommutative torus, Phys. Rev. D 63 (2001), 086001, 8 pages, hep-th/0010101.

[2] Boca F.P., Projections in rotation algebras and theta functions, Comm. Math. Phys. 202 (1999), 325-357, math.OA/9803134.

[3] Connes A., $C^{*}$ algèbres et géométrie différentielle, C. R. Acad. Sci. Paris Sér. A-B 290 (1980), A599-A604, hep-th/0101093.

[4] Connes A., Noncommutative geometry, Academic Press, Inc., San Diego, CA, 1994.

[5] Connes A., Douglas M.R., Schwarz A., Noncommutative geometry and matrix theory: compactification on tori, J. High Energy Phys. 1998 (1998), no. 2, 003, 35 pages, hep-th/9711162.

[6] Eckstein M., Anomalies in noncommutative geometry, Master's Thesis, Jagellonian University, 2010.

[7] Elliott G.A., Evans D.E., The structure of the irrational rotation $C^{*}$-algebra, Ann. of Math. 138 (1993), $477-501$.

[8] Exel R., Loring T.A., Invariants of almost commuting unitaries, J. Funct. Anal. 95 (1991), 364-376. 
[9] Frohman C., Gelca R., Skein modules and the noncommutative torus, Trans. Amer. Math. Soc. 352 (2000), 4877-4888, math.QA/9806107.

[10] Gracia-Bondía J.M., Várilly J.C., Figueroa H., Elements of noncommutative geometry, Birkhäuser Advanced Texts, Birkhäuser Boston, Inc., Boston, MA, 2001.

[11] Khalkhali M., Basic noncommutative geometry, EMS Series of Lectures in Mathematics, European Mathematical Society (EMS), Zürich, 2009.

[12] Krajewski T., Schnabl M., Exact solitons on non-commutative tori, J. High Energy Phys. 2001 (2001), no. 8, 002, 22 pages, hep-th/0104090.

[13] Landi G., Lizzi F., Szabo R.J., Matrix quantum mechanics and soliton regularization of noncommutative field theory, Adv. Theor. Math. Phys. 8 (2004), 1-82, hep-th/0401072.

[14] Luef F., Projections in noncommutative tori and Gabor frames, Proc. Amer. Math. Soc. 139 (2011), 571-582, arXiv:1003.3719.

[15] Perrot D., Anomalies and noncommutative index theory, in Geometric and Topological Methods for Quantum Field Theory, Contemp. Math., Vol. 434, Amer. Math. Soc., Providence, RI, 2007, 125-160, hepth/0603209.

[16] Pimsner M., Voiculescu D., Exact sequences for $K$-groups and Ext-groups of certain cross-product $C^{*}$ algebras, J. Operator Theory 4 (1980), 93-118.

[17] Rieffel M.A., $C^{*}$-algebras associated with irrational rotations, Pacific J. Math. 93 (1981), 415-429.

[18] Rieffel M.A., The cancellation theorem for projective modules over irrational rotation $C^{*}$-algebras, Proc. London Math. Soc. 47 (1983), 285-302.

[19] Rieffel M.A., Projective modules over higher-dimensional noncommutative tori, Canad. J. Math. 40 (1988), 257-338.

[20] Seiberg N., Witten E., String theory and noncommutative geometry, J. High Energy Phys. 1999 (1999), no. 9, 032, 93 pages, hep-th/9908142.

[21] Várilly J.C., An introduction to noncommutative geometry, EMS Series of Lectures in Mathematics, European Mathematical Society (EMS), Zürich, 2006. 